\title{
PROJECTIONS ON TENSOR PRODUCT SPACES
}

BY

\author{
E. J. HALTON ${ }^{1}$ AND W. A. LIGHT
}

\begin{abstract}
S, \Sigma, \mu),(T, \Theta, \nu)$ are finite, nonatomic measure spaces. $G$ and $H$ are finite-dimensional subspaces of $L_{1}(S)$ and $L_{1}(T)$ respectively. Both $G$ and $H$ contain the constant functions. It is shown that the relative projection constant of $L_{1}(S) \otimes H+G \otimes L_{1}(T)$ in $L_{1}(S \times T)$ is at least 3 .
\end{abstract}

I. Introduction. Suppose $X$ is a normed linear space with subspace $Y$. A projection $P: X \rightarrow Y$ is a linear transformation whose range is $Y$ and which acts as the identity on $Y$. We set $\lambda(Y, X)=\inf \{\|P\|: P$ is a projection from $X$ onto $Y$ \}. This number is called the relative projection constant of $Y$ in $X$. If we can find a projection from $X$ onto $Y$ whose norm if $\lambda(Y, X)$, then this projection is called minimal. Computing $\lambda(Y, X)$ even for quite "small" $Y$ is usually a very difficult task, and the best that can be done in most cases is to provide bounds.

In this area some recent work has focused attention on bivariate function spaces. In particular several authors have considered the cases $X=C(S \times T), Y=$ $C(S)+C(T)$ or $X=L_{p}(S \times T), Y=L_{p}(S)+L_{p}(T)$ (see [3, $\mathbf{4}$ and 6] for details). Recently, Cheney and Franchetti [1] improved the results of [6] by showing that if $X=C(S \times T)$ and $Y=C(S) \otimes H+G \otimes C(T)$, where $G$ and $H$ are finite-dimensional subspaces of $C(S)$ and $C(T)$ respectively and each containing the constant functions, then $\lambda(Y, X) \geq 3$. Jameson and Pinkus [6] had previously showed that if $G$ and $H$ are just spanned by the constant functions, then $\lambda(Y, X)=3$.

In this paper we shall take $G$ as an $n$-dimensional subspace of $L_{1}(S)$ or $L_{\infty}(S)$ spanned by $\left\{g_{1}, \ldots, g_{n}\right\}$. $H$ will be an $m$-dimensional subspace of $L_{1}(T)$ or $L_{\infty}(T)$ spanned by $\left\{h_{1}, \ldots, h_{m}\right\}$. Both $G$ and $H$ will be assumed to contain the functions which are constant almost everywhere with respect to the appropriate measure. $S$ and $T$ will be finite, nonatomic measure spaces. We shall show that $\lambda(Y, X) \geq 3$ for $X=L_{1}(S \times T), Y=L_{1}(S) \otimes H+G \otimes L_{1}(T)$.

II. Preliminary results. In this section we shall deal entirely with $L_{1}(S \times T)$. However, we shall be able to derive the same results in $L_{\infty}(S \times T)$. We shall indicate at the end of this section the minor differences that arise in $L_{\infty}(S \times T)$. So let us take $X=L_{1}(S \times T), U=L_{1}(S) \otimes H, V=G \otimes L_{1}(T)$ and $W=U+V$. We shall also assume without loss of generality that $(S, \Sigma, \mu)$ and $(T, \Theta, \nu)$ are such that $\mu(S)=\nu(T)=1$. For $x \in X$ the sections $x_{s}, x_{t}$ are defined by $x_{s}(t)=x(s, t)$ and $x_{t}(s)=x(s, t)$ for almost all $(s, t)$ belonging to $S \times T$. Unadorned norm symbols will denote $L_{1}$-norms. Thus, for example,

$$
\|u\|=\int|u(s)| d \mu, \quad u \in L_{1}(S) .
$$

Received by the editors December 19, 1983.

1980 Mathematics Subject Classification. Primary 41 A65, 41A50.

${ }^{1}$ Supported by the Science and Engineering Research Council of Great Britain.

(C) 1985 American Mathematical Society $0002-9947 / 85 \$ 1.00+\$ .25$ per page 
The $L_{\infty}$-norms are written with a subscript, as

$$
\|f\|_{\infty}=\operatorname{ess} \sup |f(s, t)|, \quad f \in L_{\infty}(S \times T) .
$$

We begin with an elementary lemma whose proof can be found in [5], for example.

LEMMA 2.1. (i) There exists a constant $\beta$ such that each element $w$ of $W$ has a representation $w=u+v$, where $u \in U, v \in V$ and $\|u\|+\|v\| \leq \beta\|w\|$.

(ii) There exists a constant $\gamma$ such that for all $x_{i} \in L_{1}(T),\left\|x_{i}\right\| \leq \gamma\left\|\sum_{1}^{n} x_{i} g_{i}\right\|$.

Here the constant $\beta$ depends only on $U$ and $V$ while the constant $\gamma$ depends only on the choice of basis for $G$.

It is well known (see $[\mathbf{2}$, p. 308]) that any finite nonatomic measure space can be written as the union of a finite number of disjoint sets each with measure no more than some prescribed tolerance $\varepsilon$. This fact can be used to show that $S$ and $T$ may be partitioned into sets $S_{i}$ and $T_{i}$, where $1 \leq i \leq k$ and $\mu\left(S_{i}\right)=\mu\left(T_{j}\right)=1 / k$. In outline this is done as follows. Firstly, partition $S$ into a finite number of disjoint sets of length at most $1 / 2 k$. By taking unions if necessary we may assume that $1 / 2 k \leq \mu\left(A_{1}\right) \leq 1 / k$. Partition $S \backslash A_{1}$ into sets of measure at most $1 / 4 k$. By taking the union of as many of these sets as necessary together with $A_{1}$ we obtain $A_{2}$ with $3 / 4 k \leq \mu\left(A_{2}\right) \leq 1 / k$. This defines the first two members of a sequence of sets $A_{1}, A_{2}, A_{3}, \ldots$. Now setting $S_{1}=\bigcup_{n=1}^{\infty} A_{n}$ gives $S_{1}$ with measure $1 / k$ as required. Further members in the partition are produced by applying this technique to $S \backslash S_{1}$ and so on. With the aid of these partitions we define functionals $E_{i} \in\left[L_{1}(S)\right]^{*}$ and $F_{j} \in\left[L_{1}(T)\right]^{*}$ by

$$
\begin{array}{ll}
E_{i} u=\int_{S_{i}} u d \mu, & u \in L_{1}(S), 1 \leq i \leq k, \\
F_{j} v=\int_{T_{j}} v d \nu, & v \in L_{1}(T), 1 \leq j \leq k .
\end{array}
$$

Now define functions $x_{i} \in L_{1}(S)$ and $y_{i} \in L_{1}(T)$ by

$$
x_{i}=k \chi_{S_{i}}, \quad y_{i}=k \chi_{T_{i}}, \quad 1 \leq i \leq k .
$$

Here $\chi_{S_{i}}$ denotes the characteristic function of the set $S_{i}$. Since $\left\{S_{i}\right\}$ is a partition of $S$ we have $\sum_{i=1}^{k} x_{i}=k$ and similarly $\sum_{i=1}^{k} y_{i}=k$. We shall set $z_{i j}=x_{i} y_{j}$, $1 \leq i, j \leq k$. It is clear that

$$
\left\|E_{i}\right\|=\left\|F_{i}\right\|=\left\|x_{i}\right\|=\left\|y_{i}\right\|=1, \quad 1 \leq i \leq k,
$$

and

$$
E_{i}\left(x_{j}\right)=F_{i}\left(y_{j}\right)=\delta_{i j}, \quad 1 \leq i, j \leq k .
$$

We also define $D_{a b} \in\left[L_{1}(S \times T)\right]^{*}$ by $D_{a b} z=E_{a}\left(F_{b} z_{s}\right)$ for all $z \in L_{1}(S \times T)$ and $1 \leq a, b \leq k$.

LEMMA 2.2. Let $P$ be a projection from $L_{1}(S \times T)$ onto $W$. Then we have

$$
\|P\| \geq \frac{1}{k^{2}} \sum_{a, b=1}^{k} \sum_{i, j=1}^{k}\left|\left(D_{a b}\right)\left(P z_{i j}\right)\right| .
$$

PROOF. We have for any $z \in L_{1}(S \times T)$ with $\|z\|=1$,

$\|P\| \geq\|P z\|=\sup \left\{\iint \phi \cdot P z d \sigma: \phi\right.$ belongs to the unit ball in $\left.L_{\infty}(S \times T)\right\}$. 
Now since the functionals $D_{a b}, 1 \leq a, b \leq k$, have disjoint support and are of unit norm we may write

$$
\|P\| \geq \sum_{a, b=1}^{k}\left|\left(D_{a b}\right)(P z)\right| \text { or }\|P\| \geq \sum_{a, b=1}^{k}\left|\left(D_{a b}\right)\left(P z_{i j}\right)\right|
$$

Summing each side of this inequality over $1 \leq i, j \leq k$ gives

$$
k^{2}\|P\| \geq \sum_{a, b=1}^{k} \sum_{i, j=1}^{k}\left|\left(D_{a b}\right)\left(P z_{i j}\right)\right|
$$

which is equivalent to the required result.

LEMMA 2.3. For any projection $P$ from $L_{1}(S \times T)$ onto $W$ we have

$$
\|P\| \geq 3-\frac{2}{k^{2}} \sum_{a, b=1}^{k}\left(D_{a b}\right)\left(P z_{a b}\right)
$$

Proof. Consider first

$$
\sum_{i=1}^{k}\left(D_{a b}\right)\left(P z_{i j}\right)=\left(D_{a b}\right)\left(P \sum_{i=1}^{k} z_{i j}\right)=\left(D_{a b}\right)\left(P\left(k y_{j}\right)\right)=\left(D_{a b}\right)\left(k y_{j}\right)=\delta_{b j} \text {. }
$$

Similarly, $\sum_{j=1}^{k}\left(D_{a b}\right)\left(P z_{i j}\right)=\delta_{a i}$. This allows us to reason as follows:

$$
\begin{aligned}
& k^{2}\|P\| \geq \sum_{a, b=1}^{k} \sum_{i, j=1}^{k}\left|\left(D_{a b}\right)\left(P z_{i j}\right)\right| \\
& \geq \sum_{a, b=1}^{k}\left[-\sum_{\substack{i, j=1 \\
i \neq a, j \neq b}}^{k}\left(D_{a b}\right)\left(P z_{i j}\right)+\sum_{j=1}^{k}\left(D_{a b}\right)\left(P z_{a j}\right)\right. \\
& \left.+\sum_{i=1}^{k}\left(D_{a b}\right)\left(P z_{i b}\right)-\left(D_{a b}\right)\left(P z_{a b}\right)\right] \\
& =\sum_{a, b=1}^{k}\left[-\sum_{i, j=1}^{k}\left(D_{a b}\right)\left(P z_{i j}\right)+2 \sum_{j=1}^{k}\left(D_{a b}\right)\left(P z_{a j}\right)\right. \\
& \left.+2 \sum_{i=1}^{k}\left(D_{a b}\right)\left(P z_{i b}\right)-2\left(D_{a b}\right)\left(P z_{a b}\right)\right] \\
& =\sum_{a, b=1}^{k}\left(-1+2 \delta_{a a}+2 \delta_{b b}-2\left(D_{a b}\right)\left(P z_{a b}\right)\right) \\
& =3 k^{2}-2 \sum_{a, b=1}^{k}\left(D_{a b}\right)\left(P z_{a b}\right) \text {. }
\end{aligned}
$$


We conclude this section as promised, with a brief discussion of the $L_{\infty}$-case. Lemma 2.3 is really not dependent on the norm employed so much as the closedness of $U+V$. We define $E_{i}, F_{i}, x_{i}, y_{i}, 1 \leq i \leq k$, as before but now $\left\|E_{i}\right\|=\left\|F_{i}\right\|=$ $1 / k$ and $\left\|x_{i}\right\|=\left\|y_{i}\right\|=k, 1 \leq i \leq k$. We continue to have the biorthogonality property. The change of scaling does not affect Lemma 2.2 and since Lemma 2.3 is an algebraic result resting only on the biorthogonality conditions we continue to have the following result.

LEMMA 2.4. Let $P$ be any projection from $L_{\infty}(S \times T)$ onto $L_{\infty}(S) \otimes H+G \otimes$ $L_{\infty}(T)$, where $H$ is finite dimensional in $L_{\infty}(T), G$ is finite dimensional in $L_{\infty}(S)$ and $G$ and $H$ contain the functions which are constant almost everywhere on $S$ and $T$ respectively. Then

$$
\|P\| \geq 3-\frac{2}{k^{2}} \sum_{a, b=1}^{k}\left(D_{a b}\right)\left(P z_{a b}\right) .
$$

III. Main theorem. With the aid of the results in the previous section we can now deduce the required result in $L_{1}(S \times T)$.

THEOREM 3.1. Let $G, H$ be finite-dimensional subspaces of $L_{1}(S)$ and $L_{1}(T)$ respectively such that they each contain the functions which are constant almost everywhere with respect to the appropriate measure. Then

$$
\lambda\left(L_{1}(S) \otimes H+G \otimes L_{1}(T), L_{1}(S \times T)\right) \geq 3 .
$$

PROOF. By Lemma 2.1 we can express $P z_{a b}$ as

$$
P z_{a b}=u_{a b}+v_{a b}=\sum_{i=1}^{n} x_{i}^{a . b} g_{i}+\sum_{j=1}^{m} y_{j}^{a, b} h_{j},
$$

where $\left\|u_{a b}\right\|+\left\|v_{a b}\right\| \leq \beta\left\|P z_{a b}\right\|$. Then

$$
\begin{aligned}
\sum_{a, b=1}^{k}\left(D_{a b}\right)\left(P z_{a b}\right) & =\sum_{a, b=1}^{k}\left[\sum_{i=1}^{n} E_{a}\left(g_{i}\right) F_{b}\left(x_{i}^{a, b}\right)+\sum_{j=1}^{m} E_{a}\left(y_{j}^{a, b}\right) F_{b}\left(h_{j}\right)\right] \\
& \leq \sum_{a, b=1}^{k}\left[\sum_{i=1}^{n}\left|E_{a}\left(g_{i}\right)\right|\left|F_{b}\left(x_{i}^{a, b}\right)\right|+\sum_{j=1}^{m}\left|E_{a}\left(y_{j}^{a, b}\right)\right|\left|F_{b}\left(h_{j}\right)\right|\right] .
\end{aligned}
$$

Now by Lemma 2.1 again we may argue as follows

$$
\left|F_{b}\left(x_{i}^{a, b}\right)\right| \leq\left\|x_{i}^{a, b}\right\| \leq \gamma\left\|\sum_{i=1}^{n} x_{i}^{a, b} g_{i}\right\| \leq \beta \gamma\left\|P z_{a b}\right\| \leq \beta \gamma\|P\| .
$$

Similarly, $\mid E_{a}\left(y_{i}^{a, b}\right) \leq \beta \delta\|P\|$, where $\delta$ is an appropriate constant depending only on the basis chosen for $H$. Hence, there exists a real number $R>0$ such that

$$
\sum_{a, b=1}^{k}\left(D_{a b}\right)\left(P z_{a b}\right) \leq R\|P\| \sum_{a, b=1}^{k}\left[\sum_{i=1}^{n}\left|E_{a}\left(g_{i}\right)\right|+\sum_{j=1}^{m}\left|F_{b}\left(h_{j}\right)\right|\right] \text {. }
$$

Now by our definition of $E_{a}, F_{b}, 1 \leq a, b \leq k$, we have

$$
\sum_{a=1}^{k}\left|E_{a}\left(g_{i}\right)\right| \leq\left\|g_{i}\right\|, \quad \sum_{b=1}^{k}\left|F_{b}\left(h_{j}\right)\right| \leq\left\|h_{j}\right\|
$$


and so

$$
\sum_{a, b=1}^{k}\left(D_{a b}\right)\left(P z_{a b}\right) \leq k R\|P\|\left[\sum_{i=1}^{n}\left\|g_{i}\right\|+\sum_{j=1}^{m}\left\|h_{j}\right\|\right] .
$$

Hence,

$$
\begin{aligned}
\|P\| & \geq 3-\frac{2}{k^{2}} \sum_{a, b=1}^{k}\left(D_{a b}\right)\left(P z_{a b}\right) \\
& \geq 3-\frac{2}{k} R\|P\|\left[\sum_{i=1}^{n}\left\|g_{i}\right\|+\sum_{j=1}^{m}\left\|h_{j}\right\|\right] .
\end{aligned}
$$

Since this inequality holds for all natural numbers $k$, we have the required result.

\section{REFERENCES}

1. E. W. Cheney and C. Franchetti, Minimal projections in tensor product spaces (Center for Numerical Analysis Report No. 184, Univ. of Texas at Austin, Austin, Texas); J. Approx. Theory (to appear).

2. N. Dunford and J. T. Schwartz, Linear operators, Part I, Interscience, New York, 1957.

3. E. J. Halton and W. A. Light, Minimal projections in bivariate function spaces, J. Approx. Theory (to appear).

4. __ Minimal projections in $L_{p}$-spaces, Univ. of Lancaster Math. Dept. Report, Lancaster, England, Sept. 1983.

5. S. M. Holland, W. A. Light and L. J. Sulley, On proximinality in $L_{1}(T \times S)$, Proc. Amer. Math. Soc. 86 (1982), 279-282.

6. G. J. O. Jameson and A. Pinkus, Positive and minimal projections in function spaces, J. Approx. Theory 37 (1983), 182-195.

Department of MAThematics, UNiversity OF LANCASTER, LANCASTER LA1 4 YL. ENGLAND 